\title{
Regulation of blood pressure by the central nucleus of the amygdala in dynamically changing appetitive and aversive classical conditioning tasks in rats
}

Ko Yamanaka ( $\nabla$ k-yamana@juntendo.ac.jp)

Department of Physiology, Graduate School of Health and Sports Science, Juntendo University, Inzai, Chiba, 270-1695, Japan

Hidefumi Waki

Juntendo University

\section{Research Article}

Keywords: blood pressure, heart rate, classical conditioning, central nucleus of the amygdala, rat

Posted Date: March 17th, 2021

DOl: https://doi.org/10.21203/rs.3.rs-109253/v2

License: (c) (i) This work is licensed under a Creative Commons Attribution 4.0 International License.

Read Full License 


\section{Abstract}

In an environment of dynamically changing conditions, humans and animals can determine whether a current situation is favorable to them and accordingly select actions. Autonomic cardiovascular tuning is as important as motor control for this function. However, neuronal mechanisms underlying the dynamic adjustments of autonomic cardiovascular responses remain unclear. In this study, we hypothesized that the amygdala plays a role in autonomic cardiovascular tuning in a dynamically changing situation. We recorded the blood pressures and heart rates of head-restrained rats during appetitive and aversive classical conditioning tasks. Rats learned varying associations between conditioned stimuli and unconditioned stimuli in appetitive, neutral, and aversive blocks. Blood pressure and heart rate in the appetitive block gradually increased in response to reward-predicting cue, preceded by a vigorously increased response to the actual reward. The predictive response was significantly higher than the responses in the neutral and aversive condition blocks. Blood pressure and heart rate responses to the air puff-predicting cue in the aversive block were significantly lower than that of the responses in the neutral block. The conditioned blood pressure response rapidly changed through condition switching. Furthermore, bilateral pharmacological inactivation of the central nucleus of the amygdala has significantly decreased reward-predictive pressor responses in the latter phase, but not in the initial phase of block change. These results suggest that blood pressure is adaptively tuned by positive and negative conditioned stimuli and that the central nucleus of the amygdala likely assists in maintaining pressor response in dynamically changing situations.

\section{New And Noteworthy}

Proper autonomic control is necessary for appropriate action selection, but neuronal mechanisms for these functions remain to be determined. Here, we demonstrated characteristic blood pressure responses to reward-predictive and aversion-predictive cues through dynamically changing conditioning tasks. Pharmacological inactivation of the central nucleus of the amygdala (CeA) has significantly attenuated reward-predictive pressor response. Thus, autonomic blood pressure is adaptively tuned by positive and negative emotional stimuli, and CeA may contribute to such adaptation.

\section{Introduction}

Predicting outcomes and preparing actions for future events based on current situation can often result in getting rewards ahead of competitors and flight from danger. Such prediction is critical for survival, especially in a dynamically changing environment. Surely motor control is essential for appropriate and rapid action, and autonomic cardiovascular tuning is likewise critical to supply energy to active skeletal muscles. However, the neuronal mechanisms underlying dynamic adjustments of autonomic cardiovascular responses are still unclear.

Previous studies show that the amygdala plays an essential role in emotional processing and defensive behavioral responses. The amygdala is classically believed to play a central role in a negative emotional 
aspect (Hilton and Zbrozyna 1963; Kapp et al. 1992; Pascoe and Kapp 1985); however, nuclei involvement in emotional, attentional, and learning processing from a positive aspect has also been reported in rodents (Calu et al. 2010; Everitt et al. 2003; Holland and Gallagher 1999; Kim et al. 2016; Roesch et al. 2012), macaques(Baxter and Murray 2002; Paton et al. 2006), and humans (Phelps and LeDoux 2005). Previous studies showed that the neuronal activities of the amygdala during appetitive and aversive conditioned stimuli are similar and correlated with autonomic arousal, such as changes in the blood pressure, suggesting an emotional arousal coding in the amygdala (Shabel and Janak 2009; Shabel et al. 2011). We previously showed that electrical and chemical stimulations of the central nucleus of the amygdala (CeA) in anesthetized rats induced bidirectional (facilitatory or inhibitory) cardiovascular responses in a region-specific manner (Yamanaka et al. 2018). In behavioral animals, we observed a sudden increase in the blood pressure immediately before exhaustion during high-intensity treadmill running with negative emotion and strong c-Fos expression in the amygdala (Kim et al. 2020). Conditioned cardiovascular responses are classically recorded during anticipation of either appetitive or aversive outcomes (Cohen and Obrist 1975; Harris and Brady 1974). Because many of these studies have been conducted in free-moving animals, it is difficult to control animal behaviors such as approaching, freezing and fleeing. Furthermore, when facing an environment where emotional context dynamically changes, it has not been demonstrated how autonomic cardiovascular response regulates and whether the amygdala is involved in the process.

Thus, we hypothesized in this study that the amygdala plays a role in autonomic cardiovascular tuning in accordance with the context of emotional arousal. In our experiments, we recorded blood pressure and heart rate from head-restrained rats to test the hypothesis during dynamically changing appetitive and aversive classical conditioning tasks and examined the effects of amygdala manipulation.

\section{Materials And Methods}

\section{Animals}

A total of 7 male Wistar-Kyoto rats (age: 7 weeks, weight: $235 \pm 44 \mathrm{~g}$ at the time of first surgery) were used in this study (Japan SLC, Inc., Japan). The animals were housed in a temperature-controlled room under a fixed 12:12 $\mathrm{h}$ dark/light cycle (6:00-18:00/18:00-6:00). Animals were provided access to food and water ad libitum until full recovery from surgery. After the start of conditional training, water access was restricted to increase motivation for sucrose rewards. Animals permitted access to water ad libitum for one day a week (weekend) but could obtain water only in the behavioral task during the rest of the week. Body weights of rats were measured every day and given a few agar blocks (containing $15 \mathrm{~mL}$ water) in their home cages when they were less than $85 \%$ of their original weights. All experiments were approved by the Ethics Committee for Animal Experiments at Juntendo University and complied with the guidelines set by the Japan Physiological Society.

\section{Surgery}


We implanted radio transmitter to record blood pressure and then head plates to fix animal's body during experiments. Recovery time after each surgery was over 1 week. During surgery, rectal temperature was monitored and maintained at $37^{\circ} \mathrm{C}$ using a heating pad (BWT-100, Bio Research Center, Japan). The level of anesthesia was checked by assessing limb withdrawal to noxious pinching. After surgery, antibiotics (benzylpenicillin, 1000U, i.m., Meiji Seika Pharma, Japan) and analgesics (meloxicam, $1 \mathrm{mg} / \mathrm{kg}$, s.c., Boehringer Ingelheim, Germany) were administered.

\section{Implantation of a transmitter for telemetry.}

A telemetric radio transmitter (HD-S10; Data Sciences International, USA) for chronic blood pressure recording from abdominal aorta was implanted, as described in previous studies(Waki et al. 2003; Yamanaka et al. 2018). Rats were anesthetized with pentobarbital sodium (50 mg/kg) by intraperitoneal (i.p.) administration and isoflurane (2.0\%-2.5\% for maintenance) using an inhalation anesthesia apparatus (Univentor 400 anesthesia unit, Univentor, Sweden). After an abdominal midline incision was made in a rat in supine position, the intestines were moved aside to allow visualization, and the abdominal aorta was carefully isolated. After a temporal blockade using a sterilized string to prevent severe blood loss, the tip of the catheter of the transmitter was inserted into the abdominal aorta along a $21 \mathrm{G}$ needle guide. The transmitter catheter was then fixed using a tissue adhesive (Vetbond, 3M, USA). The transmitter was sutured to the ventral wall of the abdominal cavity.

\section{Head-fixed operation}

All procedures for the head plate implantation (CFR-1, Narishige, Japan) were referenced to previously established studies(Kimura et al. 2012; Saiki et al. 2017; Soma et al. 2017). Rats were anesthetized with isoflurane (Pfizer, USA), 4.5\%-5.0\% for induction and 2.0\%-2.5\% for maintenance, and they were later placed on a stereotaxic frame (SR-10R-HT, Narishige, Japan). A stainless head plate was then attached to the skull using tiny stainless screw bolts (M1, 2 mm long; Yahata Neji Corporation, Japan) as anchors along with dental cements (Super-Bond C\&B, Sun Medical; Unifast II, GC Corporation, Japan).

\section{Classical conditioning task}

Rats were trained using the behavioral testing systems (Task Forcer, O'Hara and Co., Ltd., Japan) with a classical conditioning task; this task aimed to develop a behavioral model for evaluation of autonomic responses during dynamic changing cue-outcome associations with emotional arousal. Animals were fixed on a stereotaxic frame (SR-10R-HT, Narishige, Japan) in a sound-attenuated box (SAC-4201W, O'Hara and Co., Ltd., Japan) and learned varying associations between conditioned stimuli (CS) and unconditioned stimuli (US) in three types of blocks (Figs. 1A and 1B): (1) Appetitive reward (RW) block: one-tone cue (reward CS+, $10 \mathrm{kHz}, 1 \mathrm{~s}$ ) associated with sucrose delivery (reward US+; $5 \%$ sucrose, 0.08 $\mathrm{mL}$ ) and another tone cue (reward CS-, $4 \mathrm{kHz}, 1 \mathrm{~s}$ ) associated with non-reward (reward US-), (2) Aversive (AV) block: the CS+ associated with air puff (aversive US+; 30-40 psi, $1 \mathrm{~s}$ ) and the CS- associated with no air puff (aversive US-), (3) Neutral (NA) block: both CS tones predicted nothing (neutral CS+ and CS-). 
The air puff was delivered through a stainless tube placed $8-10 \mathrm{~cm}$ from the rat's face. The interval of the time between CS offset to US onset (CS-US interval) was $15 \mathrm{~s}$. The inter-trial interval was $60 \pm 15 \mathrm{~s}$. Each block consisted of 16 to 24 trials, and the order of trials (CS+ or CS- trial) was pseudo-randomly assigned. Animals could not predict the timing of block switching because none of the cues occurred with block changes. The order of blocks was fixed as RW and AV were alternatively presented; NA was then deployed in between (Fig. 1C; RW $\rightarrow \mathrm{NA} \rightarrow \mathrm{AV} \rightarrow \mathrm{NA} \rightarrow \mathrm{RW} \rightarrow \ldots$.. $\mathrm{RW}$ or AV was randomly assigned to start the daily trials. As a learning process, rats were initially trained only in the RW block. The AV context was then added, with NA context training included last. Orofacial licking movements related to reward anticipation and consumption were monitored by measuring the strain on the reward spout tube (Fig. 1A; KFG-2N-120-C1-23 amplified by DPM-911B, Kyowa, Japan).

\section{Muscimol injection}

Causality between conditioned blood pressure responses and activity of the CeA was assessed using pharmacological inactivation experiments. Four of the 7 animals were used in this experiment. Immediately before initiating the classical conditioning task of each experiment day, rats were microinjected with $\mathrm{GABA}_{\mathrm{A}}$ receptor agonist (muscimol, 80 pmol, $100 \mathrm{~nL}, \mathrm{M} 1523-10 \mathrm{MG}$, Sigma-Aldrich, USA) into the bilateral CeA ( $1.8 \mathrm{~mm}$ caudal, $3.0 \mathrm{~mm}$ lateral from bregma, and $7.0 \mathrm{~mm}$ ventral from dura) using a glass micropipette (outside diameter of 20-30 $\mu \mathrm{m}$; GC200F-10, Harvard Apparatus, USA). We injected saline (100 nL, Otsuka, Japan) to the control experiments for cardiovascular responses owing to volume effects of liquid injection. Micro-pipettes were then connected to a Hamilton microsyringe mounted on a syringe pump (LEGAT0110, KD Scientific, USA) to control the injection rate $(500 \mathrm{~nL} / \mathrm{min})$. After completing the final experiments, identification of the chemical inactivation site was confirmed by injections of fluorescent microspheres (FluoSpheres, $100 \mathrm{~nL}$, Thermo Fisher Scientific, USA) at the same coordinates stereotaxically as muscimol injection.

\section{Histology}

Rats were deeply anesthetized with sodium pentobarbital and isoflurane after completion of all experiments and intracardially perfused with saline followed by $4 \%$ paraformaldehyde (163-20145, FUJIFILM Wako Pure Chemical Corporation, Japan). The brains were then removed, post-fixed for at least $48 \mathrm{~h}$ in $4 \%$ paraformaldehyde, and replaced with $30 \%$ sucrose. Brain tissue that settled out in the sucrose solution was sliced into $50-\mu \mathrm{m}$-thick serial sections on a freezing microtome (REM-710; Yamato Kohki Industrial, Japan). The sections were then mounted on slides and imaged using a fluorescence microscope (EVOS FL Auto 2 imaging system; Thermo Fisher, USA) to map drug injection sites in the amygdala.

\section{Data analysis and statistics}


We recorded blood pressure and heart rate during the classical conditioning tasks (Fig. 2). These parameters were simultaneously recorded using the telemetry blood pressure recording system (PhysioTel, Data Sciences International, USA) with the PowerLab system (PowerLab/8s, ADInstruments, New Zealand). Mean blood pressure and heart rate were derived from pulsatile pressure signals using LabChart software (Version 8.0, AD Instruments). These data were subsequently analyzed in MATLAB (The MathWorks, USA). Artificial drops or increases in blood pressure and heart rate signals were removed and treated as missing values in the dataset. We mainly focused on our analysis on blood pressure and heart rate from CS onset to US onset (CS-US interval), and changes in the mean blood pressure ( $\triangle$ Mean blood pressure; $\triangle \mathrm{MBP})$, whereas heart rate ( $\triangle$ Heart rate; $\Delta \mathrm{HR}$ ) were calculated by subtracting mean values during baseline period 5-15 s before the CS onset. To examine the conditioning process in which animals learned the CS-US associations on each block, the magnitudes of cardiovascular responses during $6 \mathrm{~s}$ immediately before the US onset were calculated and compared using two-way analysis of variance (ANOVA) among the training sessions (day) and condition blocks (RW, NA, and AV) (Fig. 3). One-way ANOVA with Tukey-Kramer post-hoc test was used in comparing magnitudes of blood pressure and heart rate during $6 \mathrm{~s}$ immediately before the US onset among RW, NA, and AV blocks (Fig. 4). Considering that autonomic cardiovascular response is influenced by movement as well as emotion, we attempted to distinguish whether the observed cardiovascular responses were caused by the orofacial movement with anticipatory licking behavior and/or emotion induced by outcome prediction. We divided the trials into two groups based on whether the amplitude of licking was lower or higher than the threshold level (3 s.d. from the baseline) during the CS-US interval. The licking amplitude was converted into absolute values, and then the change from the baseline value was calculated for each trial $(\triangle \mathrm{abs} L \mathrm{C})$. Averaged blood pressure, heart rate, and licking movements were plotted and compared for low- and high-licking trials using one-way ANOVA with Tukey-Kramer post-hoc test (Fig. 5). Analysis of blood pressure and heart rate with switching of the context blocks was performed using ensemble responses to each CS during five trials before and after block switch (Fig. 6). Finally, we quantified and compared CS-US intervals between muscimol- and saline-injected sessions to examine the effects of inactivation of the $\mathrm{CeA}$. Average data of $\triangle \mathrm{MBP}$ in response to $\mathrm{CS}+$ for the effects of bilateral inactivation of the CeA before and after 4 trials ( 2 trials per bin) at condition block switching during the classical conditioning task were assessed. We then analyzed the data using two-way ANOVA and Mann-Whitney U-tests with trials from condition block change and drug (muscimol vs saline) factors (Fig. 7). Statistics analysis was conducted using MATLAB Statistics and Machine Learning Toolbox (The MathWorks). The criterion for statistical significance was $p=0.05$.

\section{Results}

\section{Blood pressure and heart rate responses during classical conditioning tasks}

We developed a classical conditioning task with switching among the blocks with different cue-outcome (CS-US) associations (Fig. 1). Head-restrained rats with implanted radio transmitters $(n=7)$ were trained 
to discriminate between two tone cues during appetitive reward (RW), neutral (NA), and aversive (AV) blocks. One tone $(\mathrm{CS}+, 10 \mathrm{kHz})$ cue was associated with reward delivery (reward CS+ predicts $5 \%$ sucrose, $0.08 \mathrm{~mL}$, reward US+) in the RW block, no outcome in the NA block (neutral CS+), and punishment in the AV block (aversive CS+ predicts an air puff, 30-40 psi, 1 s, aversive US+). The other tone (CS-, $4 \mathrm{kHz}$ ) cue was associated with no outcome in all condition blocks (Fig. 1B, reward, neutral, and aversive CS-). We recorded blood pressure and heart rate as physiological parameters and licking movement as a behavioral parameter during the classical conditioning task.

For example, licking movement, blood pressure, and heart rate traces in the 2 trials in each RW and AV blocks on the first day (day 1 ) and 12 days (day 12) of training are provided in Figure 2. In RW block on day 1 (Fig. 2A), licking movement, blood pressure, and heart rate were phasically increased only immediately after reward delivery (US+) but not after CS+, CS-, and US-. On day 12 (Fig. 2B), the blood pressure and heart rate gradually increased after reward $\mathrm{CS}+$, followed by a polyphasic pressor and vigorous tachycardiac response to the reward US+ but not reward CS- and US-. Conversely, in AV block on day 1 (Fig. 2C), blood pressure and heart rate increased immediately after aversive US+ (air puff). On day 12 (Fig. 2D), the blood pressure showed gradually depressive responses to aversive CS+ and polyphasic cardiovascular responses to aversive US+.

To examine how the predictive cardiovascular responses are shaped in each condition block in a dynamically changing classical conditioning task, learning curves of the mean blood pressure $(\triangle M B P)$ and heart rate $(\triangle \mathrm{HR})$ responses compared with the baseline activity during the CS-US interval were plotted from the start of learning in each condition (Fig. 3). A two-way ANOVA revealed that the mean $\triangle M B P$ value showed significant interaction of training session (1-12 days from the start of training) and condition blocks (RW, NA, and AV) in response to CS+ (Fig. 3A, $\left.F_{(22,202)}=2.42, p<0.001\right)$. There was no significant interaction in $\triangle \mathrm{MBP}$ to CS- (Fig. 3C; $F_{(22,202)}=1.03, p=0.43$ ) and $\triangle \mathrm{HR}$ responses (Fig. 3B, $C S+, F_{(22,202)}=1.11, p=0.34$; Fig. 3D, CS $\left.-, F_{(22,202)}=1.37, p=0.13\right)$. Therefore, we conducted subsequent analyses using data after sufficient training (day 7 of learning AV block) when the CS responses were stable and distinguishable.

Averaged $\triangle \mathrm{MBP}$ to reward $\mathrm{CS}+(+7.7 \pm 0.2 \mathrm{mmHg})$ and aversive $\mathrm{CS}+(-0.1 \pm 0.1 \mathrm{mmHg})$ were significantly higher and lower than that of the neutral CS $+(+0.9 \pm 0.1 \mathrm{mmHg})$, respectively (Fig. $4 \mathrm{~A}$, $F_{(2,4697)}=714.9, p<0.001$, one-way ANOVA with Tukey-Kramer post-hoc test). Similarly, $\triangle \mathrm{HR}$ to reward and aversive $\mathrm{CS}+(\mathrm{RW}: 25.9 \pm 0.8 \mathrm{bpm}, \mathrm{AV}: 12.6 \pm 0.5 \mathrm{bpm})$ was significantly higher and lower, respectively, than in the responses in NA contexts (Fig. 4B, $15.1 \pm 0.4$ bpm, $F_{(2,4702)}=115.5, p<0.001$ ). Although CS- (4 kHz tone) indicated no outcome in all trials throughout the classical conditioning tasks, both $\triangle \mathrm{MBP}$ and $\triangle \mathrm{HR}$ showed significantly different responses to reward CS- $\left(\triangle \mathrm{MBP}\right.$, Fig. $4 \mathrm{C}, F_{(2,4747)}=$ $34.3, p<0.001 ; \Delta$ HR, Fig. $\left.4 \mathrm{D}, F_{(2,4755)}=10.8, p<0.001\right)$.

Until date, we have observed predictive cardiovascular responses in condition block-dependent manner, and it is possible that the activity reflects emotional responses induced by positive and negative outcome-associated cues. However, this activity may be caused by other possible events such as 
anticipatory licking movement. To eliminate this possibility, we divided trials into 2 groups in accordance with the amplitude of licking movement during the CS-US interval being lower or higher than the threshold value ( 3 s.d. from the baseline). Even in lower-licking trials (Fig. $5 \mathrm{~A}$; totally 10,796 trials), $\triangle \mathrm{MBP}$ to reward CS $+(+4.9 \pm 0.2 \mathrm{mmHg})$ and aversive CS $+(-0.4 \pm 0.1 \mathrm{mmHg})$ were significantly higher and lower than that of the neutral CS $+(+0.2 \pm 0.1 \mathrm{mmHg})$, respectively (Fig. $5 \mathrm{~A}$, middle-left panel, $F_{(2,4989)}=$ $392.1, p<0.001$, one-way ANOVA with Tukey-Kramer post-hoc test). However, conditioned heart rate responses disappeared in lower licking trials (Fig. $5 \mathrm{~A}$ bottom panel, $F_{(2,4617)}=2.8, p>0.05$ ). Conversely,

$\triangle \operatorname{MBP}\left(F_{(2,1053)}=53.4, p<0.001\right)$ and $\operatorname{HRR}\left(F_{(2,972)}=9.4, p<0.001\right)$ responses were increased not only in the RW block but also in the NA and AV blocks in parallel with licking amplitude as in the higher licking trials (Fig. 5B; totally 1,505 trials), suggesting that the licking movement also strongly affected the cardiovascular responses. Therefore, at least for the blood pressure, it is possible that the conditiondependent characteristic responses are caused by emotional cue-outcome association, rather than by licking movement.

\section{Dynamic changes in cardiovascular responses triggered by condition block switching}

We then assessed predictive cardiovascular responses during condition block switching using our dynamically changing appetitive and aversive classical conditioning task. The pseudo-color plots of averaged $\triangle \mathrm{MBP}$ in response to $\mathrm{CS}+$ and $\mathrm{CS}-$ in 5 trials before and after block switching (Fig. 6A, $N A \rightarrow R W \rightarrow N A$; Fig. 6B, NA $\rightarrow A V \rightarrow N A$ ) indicate that unanticipated association switching from neutral to reward condition blocks $(\mathrm{NA} \rightarrow \mathrm{RW})$ produced $\triangle \mathrm{MBP}$ to reward $\mathrm{CS}+(-1.4 \pm 0.3 \mathrm{mmHg}, p=0.42$, MannWhitney U-test) similar to responses in the previous NA condition block $(-1.1 \pm 0.2 \mathrm{mmHg})$. However, once receiving reward US+, responses to reward CS+ were dramatically increased $(4.4 \pm 0.4 \mathrm{mmHg}, p<$ 0.001, Mann-Whitney U-test). Pressor responses to reward CS+ were maintained until a few trials after switching back to the neutral block (RW $\rightarrow \mathrm{NA}$ ): $4.6 \pm 0.3 \mathrm{mmHg}$ in 5 trials before block switching, $3.9 \pm 0.4$ $\mathrm{mmHg}$ in the first trial ( $p<0.001$, compared with $\triangle \mathrm{MBP}$ to CS + in NA block), $1.0 \pm 0.4 \mathrm{mmHg}$ in the second trial $(p<0.001),-0.2 \pm 0.3 \mathrm{mmHg}$ in the third trial $(p=0.005)$, and $-0.8 \pm 0.3 \mathrm{mmHg}$ in the fourth trial $(p=0.52)$ after block switching. Immediately after the transition from neutral to aversive blocks $(\mathrm{NA} \rightarrow \mathrm{AV})$, slight but significant depressor response to aversive $\mathrm{CS}+(-0.7 \pm 0.1 \mathrm{mmHg}$ in 5 trials before block switching; $-1.1 \pm 0.3 \mathrm{mmHg}$ in the first trial, $p=0.06 ;-1.2 \pm 0.2 \mathrm{mmHg}$ in the second trial, $p<$ 0.001 ) and prominent tri-phasic responses (increase-decrease-increase) to aversive US+ were observed (Fig. 6B). Cardiovascular responses to reward- and aversive-predictive cues rapidly changed, guided by reward and aversive expectations.

\section{Bilateral inactivation of the CeA attenuated reward prediction-induced pressor response}


In this study, we observed that reward and aversion prediction induced higher and lower blood pressure responses compared with that in a neutral condition, respectively, in the classical conditioning task with dynamically changed cue-outcome associations. Previously, we reported that microstimulation of the CeA induced site-specific, bidirectional (pressor and depressor) cardiovascular responses in anesthetized rats, suggesting involvement in autonomic tuning(Yamanaka et al. 2018), through supporting the expression of defensive behaviors in emergency situations with emotional arousal. If the CeA plays a causal role in the regulation of blood pressure during emotional arousal, inactivation of CeA should abolish responses to emotional CSs.

We then examined the effects of cardiovascular responses to CSs to test this possibility via inactivation of bilateral CeA (1.8 mm caudal to bregma, $3.0 \mathrm{~mm}$ lateral to midline, and $7.0 \mathrm{~mm}$ ventral to dura). The $\mathrm{GABA}_{\mathrm{A}}$ receptor agonist muscimol $(80 \mathrm{pmol}, 100 \mathrm{~nL})$ was microinjected while the rats performed the conditioning task. In total, we performed 23 injections (muscimol, 12 injections; saline, 11 injections) in 4 of the 7 rats. The injection site was confirmed using fluorescent microspheres (FluoSpheres, $100 \mathrm{~nL}$ ) based on the histology (Fig. 7; right panel). All animals were injected with both muscimol and saline in different sessions. Inactivation of bilateral CeA using muscimol compared with saline injection caused significant decreases in $\triangle \mathrm{MBP}$ before and after switching from the RW to NA blocks (Fig. 7, $F_{(15,1287)}=$ $2.64, p<0.006$, significant interaction, two-way ANOVA). Notably, the decrease in $\triangle M B P$ was observed during the second half of the RW block $(p<0.001)$ but not immediately after NA $\rightarrow$ RW switching $(p>0.05$, Mann-Whitney U-test). CeA likely plays a more important role in maintaining rather than in acquiring emotional responses to salient events.

\section{Discussion}

In this study, we demonstrated that reward-predictive and aversion-predictive cues induced pressor and depressor blood pressure responses, respectively. The characteristic autonomic responses were acquired by learning across sessions and were observed even with lower-licking trials. Switching condition block provokes rapid regulation of the blood pressure in accordance with its emotional valence. Furthermore, blockade of the activity of the CeA impaired pressor responses during switching from reward to neutral condition blocks. We demonstrate that cardiovascular responses are adaptively and rapidly tuned by positive and negative emotional stimuli, and CeA may contribute to the maintenance of adaptive regulations.

We confirmed the characteristic cardiovascular responses in our classical conditioning tasks. Blood pressure exhibited a phasic and gradual increase in response to reward-predictive cues and a phasic decrease to neutral- and aversive-predictive cues. The heart rate increased in response to cues under all conditions compared with the baseline values, but the level of tachycardia varied among the conditions: reward CS+ induced predominant tachycardia; however, aversive CS+ evoked a relatively small increase in heart rate compared with neutral CS+. These condition-dependent characteristic cardiovascular responses were progressively acquired through several sessions and consistently expressed after sufficient learning. The blood pressure responses robustly discriminated conditions, but the increase in 
heart rate disappeared in lower-licking trials. Both the blood pressure and heart rate were increased in parallel with the amplitude of licking movement. These results suggest that both blood pressure and heart rate are affected by orofacial licking movement; however, at least the blood pressure may be regulated by additional factors, except orofacial movement per se, such as emotion. Previous studies have reported cardiovascular responses during classical conditioning task using free-moving and restrained animals in several species, such as pigeon(Cohen and Durkovic 1966), rabbit(Pascoe and Kapp 1985; Powell et al. 1997), dog(Anderson and Brady 1972; Dykman and Gantt 1956; Obrist and Webb 1967), rodent(Holdstock and Schwartzbaum 1965; Iwata and LeDoux 1988; Shabel and Janak 2009; Shabel et al. 2011), marmoset(Braesicke et al. 2005; Mikheenko et al. 2010), macaque(Randall et al. 1975), and humans(Hastings and Obrist 1967; Wexler and De Leon 1979). Appetitive conditioning using food or water as a reward US, induces pressor and tachycardia during the CS-US interval, whereas, in aversive conditioning, using electrical shock as punishment US often causes pressor or tachycardia, but some bradycardic responses are reported(Cohen and Obrist 1975; Harris and Brady 1974; Randall et al. 1975). In this study, small but significant lower blood pressure responses to air puff-predicting CS+ were also observed. Air puff is definitely acting as the US based on the remarkable tri-phasic cardiovascular responses. Considering a pressor response temporally in the early stage of learning (Fig. 3A; training session "Day 3"), one possible reason that air puff-predicting CS+ caused suppression but not facilitation of cardiovascular responses includes overtraining of the classical conditioning procedures, where the animals cannot avoid punishment (e.g., learned helplessness). The reasons for the lower response level of aversive CS+ compared with that of reward CS+ is that the air puff might be a mild stimulus compared with electrical shock and/or floor effect. In the present study, we used a neutral block in which CS+ was associated with no-outcome as a different measure from the reward block. As midbrain dopamine neurons encoding reward-prediction error signal have been reported to show a decrease in their activities in response to no-reward conditions(Matsumoto and Hikosaka 2009), no-outcome conditions may have functioned as an aversive rather than a neutral measure.

Interestingly, reward CS- induced relatively higher blood pressure and lower heart rate responses, although CS- was not associated with either reward or punishment in any condition blocks. Animals could get rewarded in our task after only reward CS+. The RW block with the highest value CS+ represented higher state value, whereas CS- in the block induced stronger negative emotion compared with other condition blocks. This possibility suggests that the value of an option (CS-) is assigned relatively, rather than absolutely, and is often influenced by the value of the paired option (CS+) (Elliott et al. 2008).

The roles of the amygdala in classical conditioning have already been examined in previous studies, particularly through fear conditioning in free-moving animals. The CeA projects into various regions such as the lateral hypothalamus, the periaqueductal grey and brainstem to regulate autonomic sympathetic and parasympathetic activities and endocrine responses to promote defensive behaviors (such as attack, escape, and freeze)(Davis 1992). Lesions in the bilateral amygdala attenuated conditioned acceleration of heart rate in both appetitive(Braesicke et al. 2005) and aversive(Iwata et al. 1986; LeDoux et al. 1990; Mikheenko et al. 2010) conditioning using free-moving animals. These findings suggest that the 
amygdala may be involved in the autonomic arousal in emotional processing. Several past studies have examined either appetitive or aversive condition blocks; however, little is known concerning autonomic tuning in different conditions that changes dynamically between appetitive and aversive. Our results are also consistent with previous studies in which the amygdala (CeA) plays an important role in accelerating cardiovascular tuning. Notably, the attenuation of pressor responses by amygdala inactivation did not appear immediately after switching to the appetitive (RW) condition block but appeared when switching from the appetitive to neutral $(\mathrm{RW} \rightarrow \mathrm{NA})$ block, suggesting a more crucial role in the maintenance, than acquisition, of emotional autonomic tuning.

Lack of significant changes in the cardiovascular responses to aversive-predicting cue by amygdala inactivation is a limitation of the present study. Conditioned responses to fear-predictive stimuli are taskdependent(Harris and Brady 1974), and decelerated responses observed in our study were not sufficiently strong. In addition, based on previous electrophysiological studies, several amygdala neurons encode emotional arousal that respond to both appetitive- and aversive-predicting CS(Shabel and Janak 2009; Shabel et al. 2011). As results in the present study, we reported that CeA microstimulation induces sitespecific bidirectional (pressor and depressor) cardiovascular responses (Yamanaka et al. 2018). Blockade of the amygdala may diminish autonomic responses if accelerated or more decelerated responses are evoked by aversive CS.

Our findings demonstrated cardiovascular tuning and the involvement of the CeA in dynamically changing the CS-US associations. The characteristic blood pressure responses rapidly adapt in a few trials immediately after condition block switching. Such rapid switching is reminiscent of the activity of neurons of the amygdala(Belova et al. 2007), striatum(Lauwereyns et al. 2002), and midbrain dopamine neurons(Roesch et al. 2007). Blockade of dopamine D1 receptor in the striatum impaired contextdependent behavioral responses(Nakamura and Hikosaka 2006; Ueda et al. 2017). Artificial suppression of the midbrain dopaminergic neurons during fear-to-safety context switching induced a delay in the extinction of fear conditioning behavior(Luo et al. 2018). In addition, the activity of dopamine neurons has also been reported to contribute to the prediction of reward as well as aversion stimuli(Matsumoto and Hikosaka 2009; Moriya et al. 2018). Anatomically, the amygdala also receives dopaminergic inputs(Garris and Wightman 1995). There is a direct projection from the amygdala (basolateral region) to the striatum, whereas projection from the striatum to the amygdala is believed to be polysynaptic. Functionally, activities of the striatal neurons increased in response to reward-predicting cue, but their activities were attenuated by the blockade of the amygdala(Ambroggi et al. 2008). Based on these evidences, condition-dependent cardiovascular responses observed in the present study may reflect the differences in the outcome expectation with the functional interactions between the amygdala and striatum via dopaminergic inputs(Averbeck and Costa 2017). On the other hand, the neuronal circuits of aversion-predictive depressive responses are still unclear. Based on behavioral and autonomic aspects and functional connections to the amygdala, possible candidates are the midbrain periaqueductal gray which is involved in negative emotional processing and freezing behavior induced by fearful stimuli (Amorapanth et al. 1999; Buhle et al. 2013), and the claustrum which shows depressive responses to artificial stimulation and functionally connected with the amygdala (Kim et al. 2020). Thus, future studies 
should further examine the functions of neuronal circuits that may support and maintain the emotional expression suitable for a dynamically changing environment.

\section{Declarations}

\section{Acknowledgements}

We thank Drs. K. Nakamura, K. Enomoto and S. Nonomura for helpful comments. M. Koizumi and Y. Ueda for helping with the experimental setup. We are grateful to W. Kurosaka, G. Saito, A. Nakagawa, K. Tsukioka, and J. Kim for valuable discussion.

\section{Grants}

This work was supported by Grant-in-Aid for Scientific Research (C) 20K11396, Young Scientists (B) 16K16485, and Exploratory Research 19K22582 (to K.Y.), MEXT-Supported Program for the Strategic Research Foundation at Private Universities, 2014-2018 (to H.W.), and Private University Research Branding Project (to H.W.).

\section{Author contributions}

K.Y. and H.W. designed the research; K.Y. conducted the experiments; K.Y. analyzed the data; and K.Y. and H.W. wrote and reviewed the manuscript.

\section{Competing interests}

The authors declare no competing financial interests.

\section{References}

Ambroggi F, Ishikawa A, Fields HL, and Nicola SM. Basolateral amygdala neurons facilitate rewardseeking behavior by exciting nucleus accumbens neurons. Neuron 59: 648-661, 2008.

Amorapanth P, Nader K, and LeDoux JE. Lesions of periaqueductal gray dissociate-conditioned freezing from conditioned suppression behavior in rats. Learn Mem 6: 491-499, 1999.

Anderson DE, and Brady JV. Differential preparatory cardiovascular responses to aversive and appetitive behavioral conditioning. Cond Reflex 7: 82-96, 1972.

Averbeck BB, and Costa VD. Motivational neural circuits underlying reinforcement learning. Nat Neurosci 20: 505-512, 2017.

Baxter MG, and Murray EA. The amygdala and reward. Nat Rev Neurosci 3: 563-573, 2002. 
Belova MA, Paton JJ, Morrison SE, and Salzman CD. Expectation modulates neural responses to pleasant and aversive stimuli in primate amygdala. Neuron 55: 970-984, 2007.

Braesicke K, Parkinson JA, Reekie Y, Man MS, Hopewell L, Pears A, Crofts H, Schnell CR, and Roberts AC. Autonomic arousal in an appetitive context in primates: a behavioural and neural analysis. Eur $\mathrm{J}$ Neurosci 21: 1733-1740, 2005.

Buhle JT, Kober H, Ochsner KN, Mende-Siedlecki P, Weber J, Hughes BL, Kross E, Atlas LY, McRae K, and Wager TD. Common representation of pain and negative emotion in the midbrain periaqueductal gray. Soc Cogn Affect Neurosci 8: 609-616, 2013.

Calu DJ, Roesch MR, Haney RZ, Holland PC, and Schoenbaum G. Neural Correlates of Variations in Event Processing during Learning in Central Nucleus of Amygdala. Neuron 68: 991-1001, 2010.

Cohen DH, and Durkovic RG. Cardiac and respiratory conditioning, differenatiation, and extinction in the pigeon. J Exp Anal Behav 9: 681-688, 1966.

Cohen DH, and Obrist PA. Interactions between behavior and the cardiovascular system. Circ Res 37: 693706, 1975.

Davis M. The role of the amygdala in fear-potentiated startle: implications for animal models of anxiety. Trends Pharmacol Sci 13: 35-41, 1992.

Dykman RA, and Gantt WH. Relation of experimental tachycardia to amplitude of motor activity and intensity of the motivating stimulus. Am J Physiol 185: 495-498, 1956.

Elliott R, Agnew Z, and Deakin JF. Medial orbitofrontal cortex codes relative rather than absolute value of financial rewards in humans. Eur J Neurosci 27: 2213-2218, 2008.

Everitt BJ, Cardinal RN, Parkinson JA, and Robbins TW. Appetitive behavior: impact of amygdaladependent mechanisms of emotional learning. Ann N Y Acad Sci 985: 233-250, 2003.

Garris PA, and Wightman RM. Distinct pharmacological regulation of evoked dopamine efflux in the amygdala and striatum of the rat in vivo. Synapse 20: 269-279, 1995.

Harris AH, and Brady JV. Animal learning. Visceral and autonomic conditioning. Annu Rev Psycho/ 25: 107-133, 1974.

Hastings SE, and Obrist PA. Heart rate during conditioning in humans: effect of varying the interstimulus (CS-UCS) interval. J Exp Psychol 74: 431-442, 1967.

Hilton SM, and Zbrozyna AW. Amygdaloid region for defence reactions and its efferent pathway to the brain stem. J Physiol 165: 160-173, 1963. 
Holdstock TL, and Schwartzbaum JS. Classical conditioning of heart rate and Galvanic skin response in the rat. Psychophysiology 2: 25-38, 1965.

Holland PC, and Gallagher M. Amygdala circuitry in attentional and representational processes. Trends Cogn Sci 3: 65-73, 1999.

Iwata J, and LeDoux JE. Dissociation of associative and nonassociative concomitants of classical fear conditioning in the freely behaving rat. Behav Neurosci 102: 66-76, 1988.

Iwata J, LeDoux JE, Meeley MP, Arneric S, and Reis DJ. Intrinsic neurons in the amygdaloid field projected to by the medial geniculate body mediate emotional responses conditioned to acoustic stimuli. Brain Res 383: 195-214, 1986.

Kapp BS, Whalen PJ, Supple WF, and Pascoe JP. Amygdaloid contributions to conditioned arousal and sensory information processing. In: The amygdala: Neurobiological aspects of emotion, memory, and mental dysfunction, edited by Aggleton JPWiley-Liss, 1992, p. 229-254.

Kim J, Pignatelli M, Xu S, Itohara S, and Tonegawa S. Antagonistic negative and positive neurons of the basolateral amygdala. Nat Neurosci 2016.

Kim J, Yamanaka K, Tsukioka K, and Waki H. Potential role of the amygdala and posterior claustrum in exercise intensity-dependent cardiovascular regulation in rats. Neuroscience 432: 150-159, 2020.

Kimura R, Saiki A, Fujiwara-Tsukamoto Y, Ohkubo F, Kitamura K, Matsuzaki M, Sakai Y, and Isomura Y. Reinforcing operandum: rapid and reliable learning of skilled forelimb movements by head-fixed rodents. J Neurophysio/ 108: 1781-1792., 2012.

Lauwereyns J, Watanabe K, Coe B, and Hikosaka O. A neural correlate of response bias in monkey caudate nucleus. Nature 418: 413-417, 2002.

LeDoux JE, Cicchetti P, Xagoraris A, and Romanski LM. The lateral amygdaloid nucleus: sensory interface of the amygdala in fear conditioning. J Neurosci 10: 1062-1069, 1990.

Luo R, Uematsu A, Weitemier A, Aquili L, Koivumaa J, McHugh TJ, and Johansen JP. A dopaminergic switch for fear to safety transitions. Nat Commun 9: 2483, 2018.

Matsumoto $\mathbf{M}$, and Hikosaka $\mathbf{0}$. Two types of dopamine neuron distinctly convey positive and negative motivational signals. Nature 2009.

Mikheenko Y, Man MS, Braesicke K, Johns ME, Hill G, Agustin-Pavon C, and Roberts AC. Autonomic, behavioral, and neural analyses of mild conditioned negative affect in marmosets. Behav Neurosci 124: 192-203, 2010. 
Moriya S, Yamashita A, Kawashima S, Nishi R, Yamanaka A, and Kuwaki T. Acute aversive stimuli rapidly increase the activity of ventral tegmental area dopamine neurons in awake mice. Neuroscience 386: 1623, 2018.

Nakamura K, and Hikosaka $\mathbf{0}$. Role of dopamine in the primate caudate nucleus in reward modulation of saccades. J Neurosci 26: 5360-5369, 2006.

Obrist PA, and Webb RA. Heart rate during conditioning in dogs: relationship to somatic-motor activity. Psychophysiology 4: 7-34, 1967.

Pascoe JP, and Kapp BS. Electrophysiological characteristics of amygdaloid central nucleus neurons during Pavlovian fear conditioning in the rabbit. Behav Brain Res 16: 117-133, 1985.

Paton JJ, Belova MA, Morrison SE, and Salzman CD. The primate amygdala represents the positive and negative value of visual stimuli during learning. Nature 439: 865-870, 2006.

Phelps EA, and LeDoux JE. Contributions of the amygdala to emotion processing: from animal models to human behavior. Neuron 48: 175-187, 2005.

Powell DA, Chachich M, Murphy V, McLaughlin J, Tebbutt D, and Buchanan SL. Amygdala-prefrontal interactions and conditioned bradycardia in the rabbit. Behav Neurosci 111: 1056-1074, 1997.

Randall DC, Brady JV, and Martin KH. Cardiovascular dynamics during classical appetitive and aversive conditioning in laboratory primates. Pavlov J Biol Sci 10: 66-75, 1975.

Roesch MR, Calu DJ, and Schoenbaum G. Dopamine neurons encode the better option in rats deciding between differently delayed or sized rewards. Nat Neurosci 2007.

Roesch MR, Esber GR, Li J, Daw ND, and Schoenbaum G. Surprise! Neural correlates of Pearce-Hall and Rescorla-Wagner coexist within the brain. Eur J Neurosci 35: 1190-1200, 2012.

Saiki A, Sakai Y, Fukabori R, Soma S, Yoshida J, Kawabata M, Yawo H, Kobayashi K, Kimura M, and Isomura $Y$. In vivo spiking dynamics of intra- and extratelencephalic projection neurons in rat motor cortex. Cereb Cortex 2017.

Shabel SJ, and Janak PH. Substantial similarity in amygdala neuronal activity during conditioned appetitive and aversive emotional arousal. Proc Natl Acad Sci U S A 106: 15031-15036, 2009.

Shabel SJ, Schairer W, Donahue RJ, Powell V, and Janak PH. Similar neural activity during fear and disgust in the rat basolateral amygdala. PLoS One 6: e27797, 2011.

Soma S, Saiki A, Yoshida J, Rios A, Kawabata M, Sakai Y, and Isomura Y. Distinct laterality in forelimbmovement representations of rat primary and secondary motor cortical neurons with intratelencephalic and pyramidal tract projections. J Neurosci 37: 10904-10916, 2017. 
Ueda Y, Yamanaka K, Noritake A, Enomoto K, Matsumoto N, Yamada H, Samejima K, Inokawa H, Hori Y, Nakamura K, and Kimura M. Distinct functions of the primate putamen direct and indirect pathways in adaptive outcome-based action selection. Front Neuroanat 11: 66, 2017.

Waki H, Kasparov S, Wong LF, Murphy D, Shimizu T, and Paton JF. Chronic inhibition of endothelial nitric oxide synthase activity in nucleus tractus solitarii enhances baroreceptor reflex in conscious rats. $J$ Physio/ 546: 233-242, 2003.

Wexler HK, and De Leon G. Classical conditioning of human systolic pressure: replication and extension using three stimulus durations. Pavlov J Biol Sci 14: 20-30, 1979.

Yamanaka K, Takagishi M, Kim J, Gouraud SS, and Waki H. Bidirectional cardiovascular responses evoked by microstimulation of the amygdala in rats. J Physiol Sci 68: 233-242, 2018.

\section{Figures}

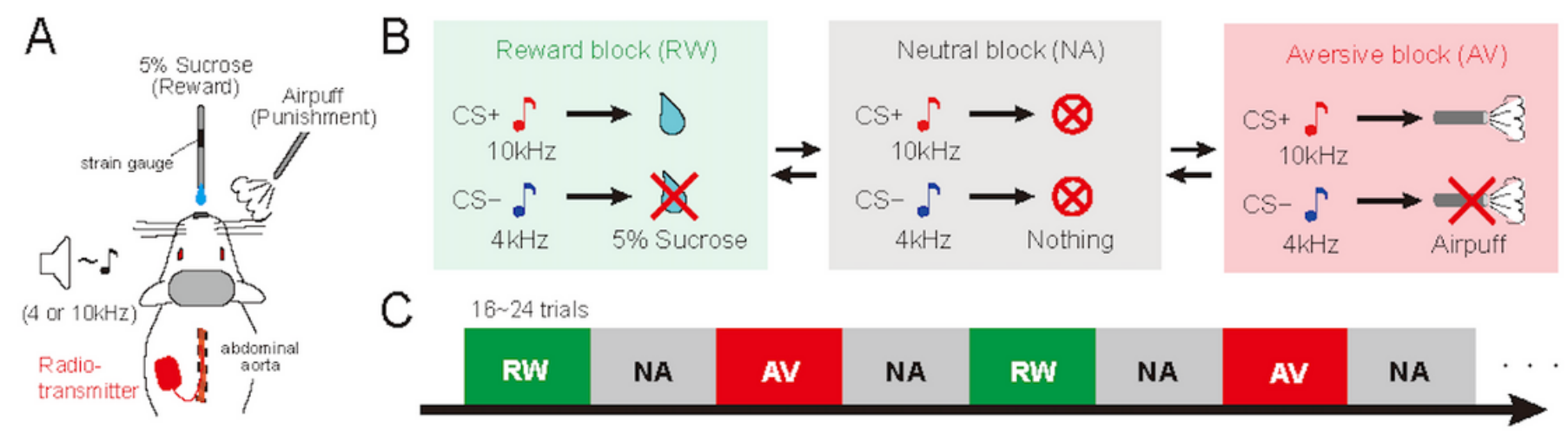

Figure 1

Classical conditioning task with emotional valence. A, Schematic diagram of the behavioral task. Stainless tubes for reward and air puff delivery were placed in the front of head-fixed rat's face. A radio transmitter was implanted into the abdominal aorta to record blood pressure by telemetry. Strain gauge was attached on the reward tube to monitor the amplitude of licking movement. B, Pavlovian procedure with three distinct context blocks. A tone cue (CS+, $10 \mathrm{kHz})$ preceded reward (US+, $5 \%$ sucrose), and another cue (CS-, $4 \mathrm{kHz}$ ) preceded non-reward (US-) in the reward (RW) blocks; CS+ preceded an air puff (US+) and the CS- preceded no-air puff (US-) in the aversive (AV) blocks; both CS tones preceded nothing in the neutral (NA) blocks. CS, conditioned stimulus; US, unconditioned stimulus. C, Trial sequence of the behavioral task. NA blocks were alternately deployed between RW and AV blocks. There were 16 to 24 trials per block. The order of blocks was fixed, but whether it started with RW or AV was randomized for every experimental day. There was no sign when changing blocks. 
A

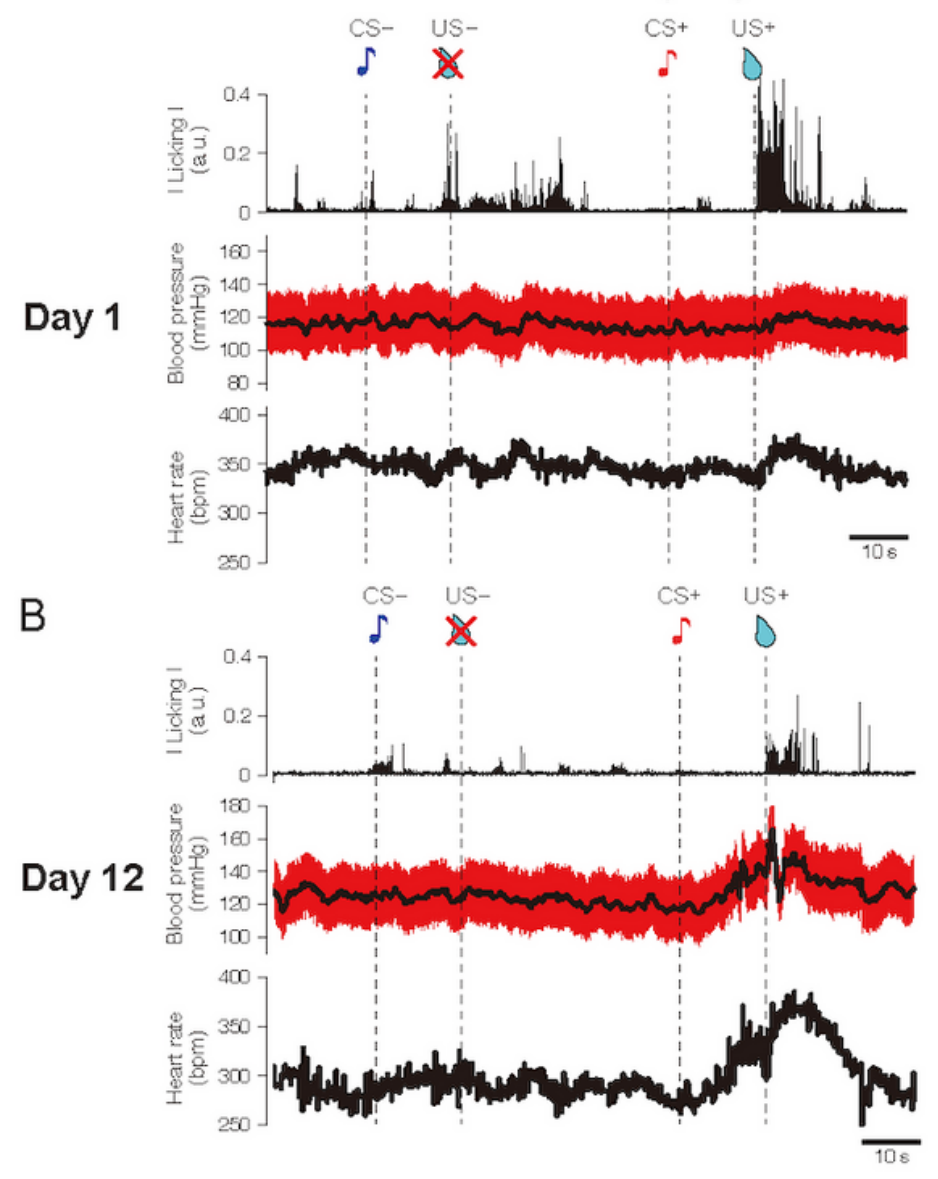

C Aversive block (AV)
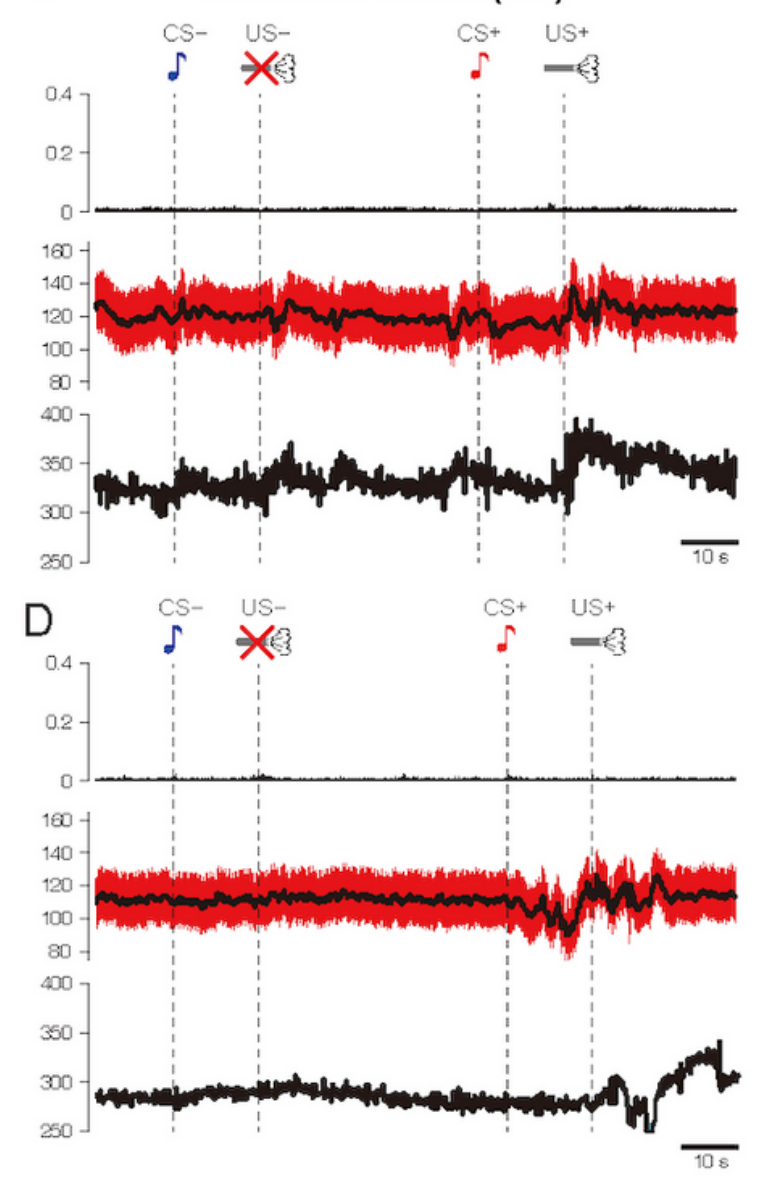

\section{Figure 2}

Blood pressure, heart rate, and licking responses during appetitive and aversive blocks in the classical conditioning task. Example traces of absolute value-converted licking (top panel), blood pressure (middle panel), and heart rate (bottom panels) in two trials during the appetitive reward (RW) context block $(A, B)$ and aversive air puff (AV) context block (C, D) on day $1(A, C)$ and day $12(B, D)$ of the Pavlovian conditioning task. Dotted vertical lines indicate the timing of CS or US onset. 


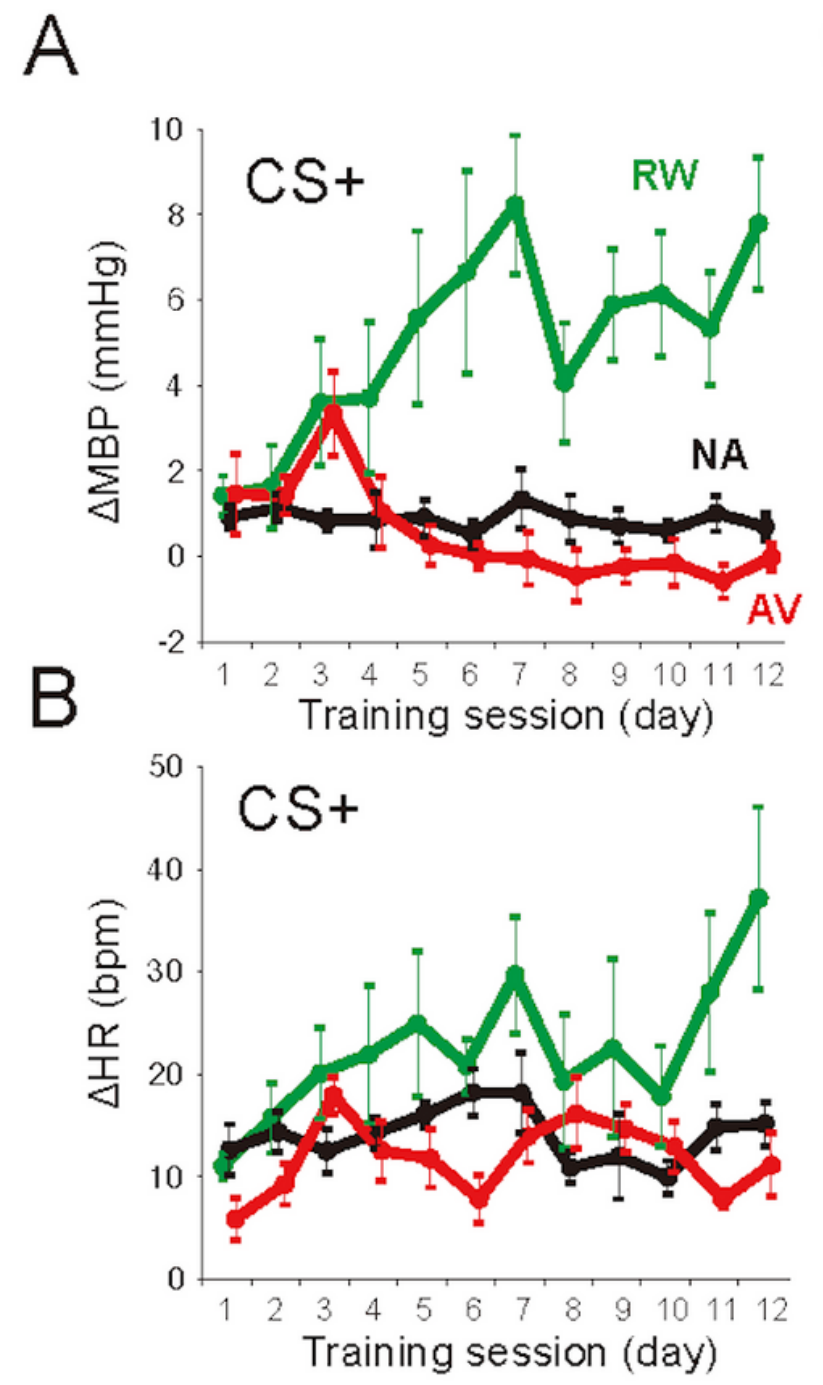

C
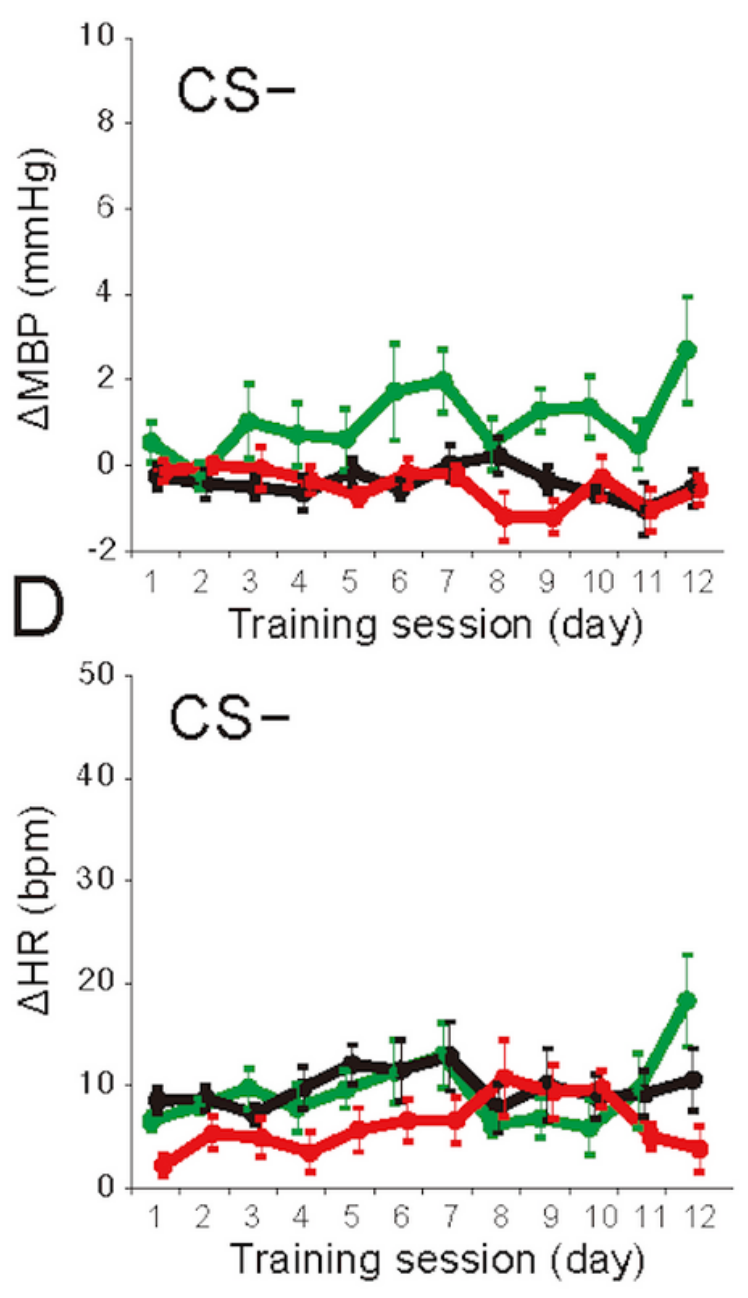

\section{Figure 3}

Learning process. A-B, Learning curves of averaged magnitude of responses of $\triangle$ mean blood pressure (MBP) (A) and $\triangle$ heart rate (HR) (B) to $C S+$ in the reward (RW; green line), neutral (NA; black line), and aversive (AV; red line) context blocks. C-D, Same as A-B, but in response to CS-. Two-way ANOVA among training session (day) and context blocks (RW, NA, and AV). 

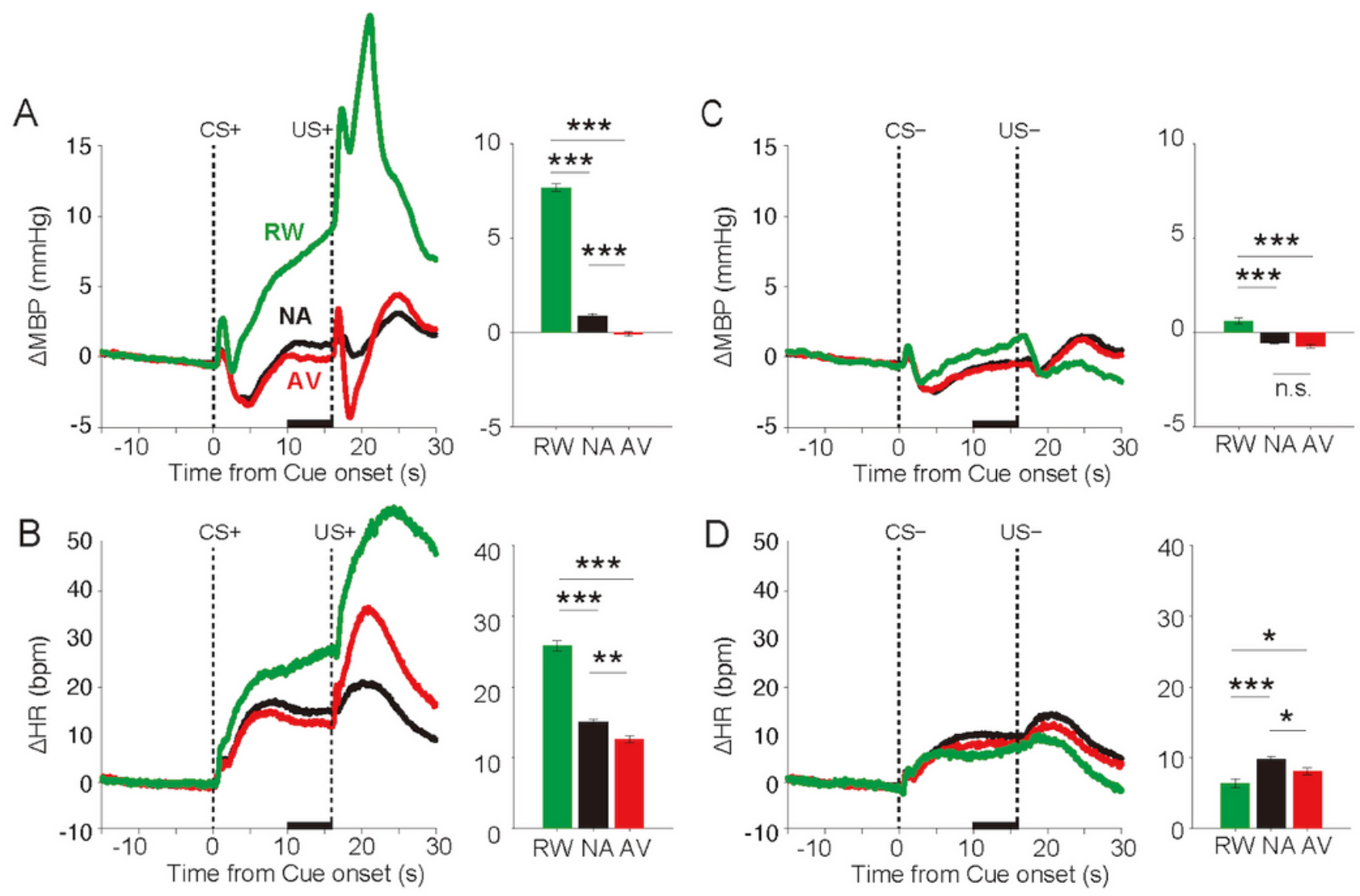

\section{Figure 4}

Blood pressure and heart rate responses during the classical conditioning task. A-B, Averaged responses of mean blood pressure $(A, \triangle M B P)$ and heart rate $(B, \triangle H R)$ responses to $C S+$ and US+ during the reward (RW; green line), neutral (NA; black line), and aversive (AV; red line) context blocks. C-D, Same as A-B, but during CS- and US-. ${ }^{*} p<0.05,{ }^{* *} p<0.001$, one-way ANOVA with Bonferroni's post hoc test. 


\section{A Lower licking trials (total 10,796 trials)}
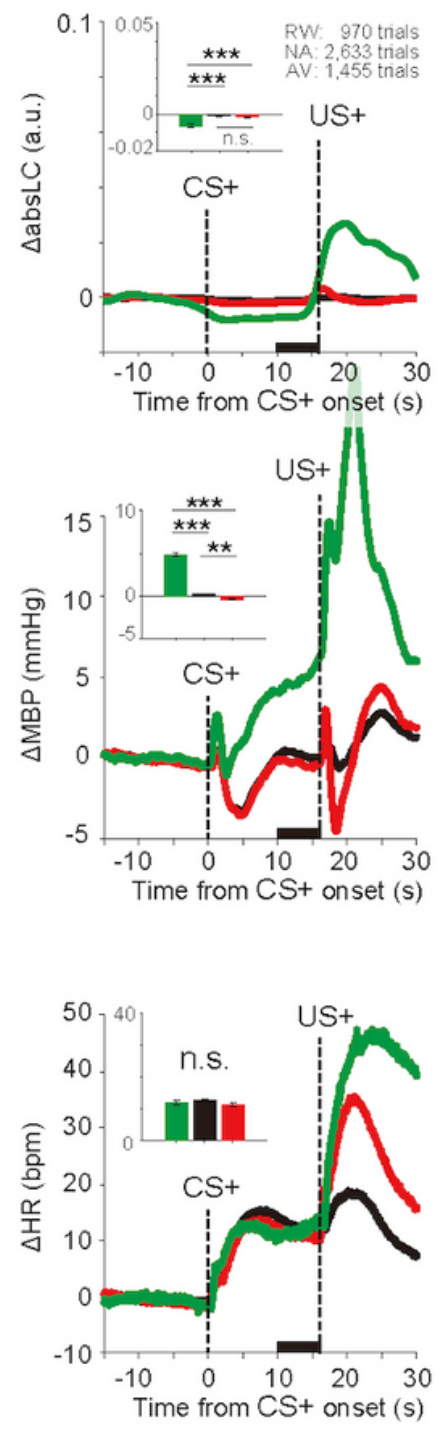
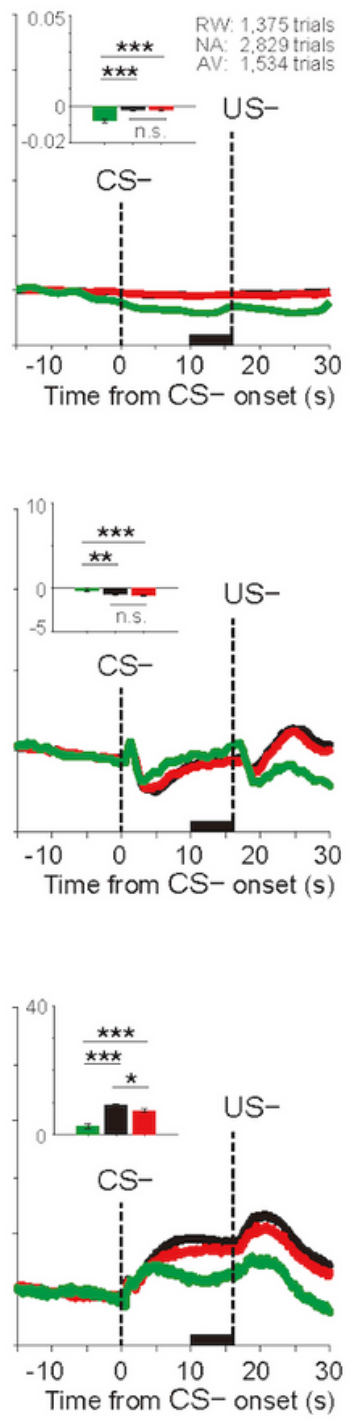

B Higher licking trials
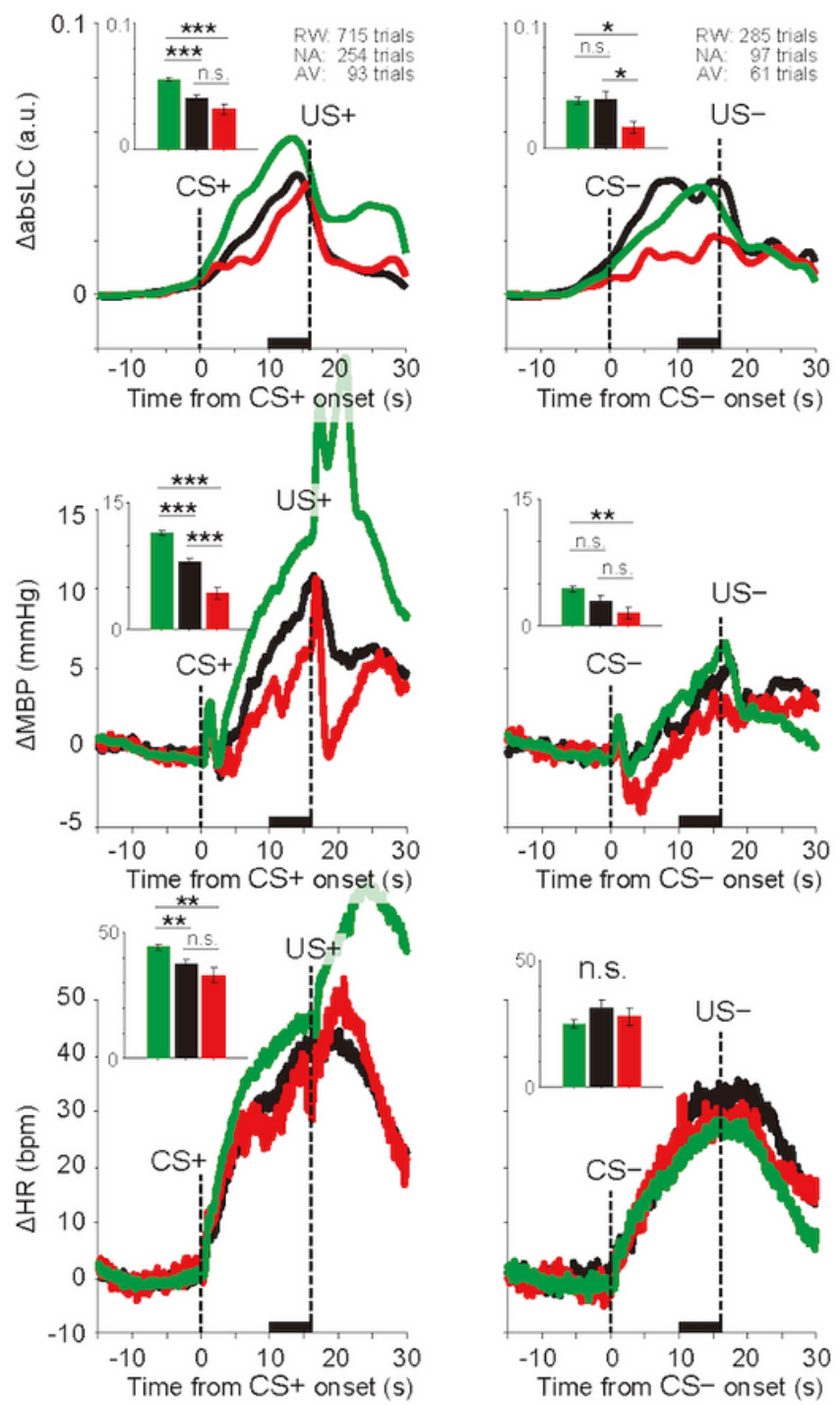
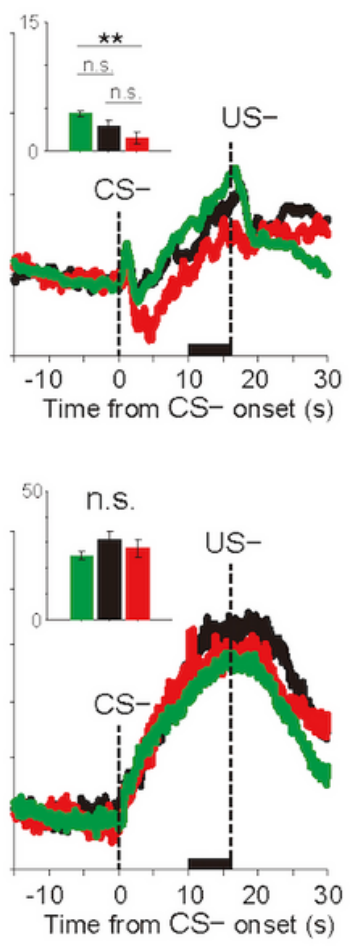

Figure 5

Effects of orofacial licking movement to cardiovascular responses. Averaged responses and quantitative bar graphs (inset of each figure) of licking ( $\triangle \mathrm{absLC}$, top panels), mean blood pressure ( $\triangle \mathrm{MBP}$, middle panels), heart rate $(\triangle \mathrm{HR}$, bottom panels), and responses to $C S+$ (left panels) and CS- (right panels) during the reward (RW, green line and bar), neutral (NA, black line and bar), and aversive (AV, red line and bar) context blocks in trials with the amplitude of licking was lower (A, totally 10,796 trials) or higher (B, higher licking trials, totally 1,505 trials) than the threshold (3 s.d. from baseline) during CS-US interval. ${ }^{*} p$ $<0.05,{ }^{\star *} p<0.01,{ }^{\star \star *} p<0.001$, one-way ANOVA with Bonferroni's post hoc test. 


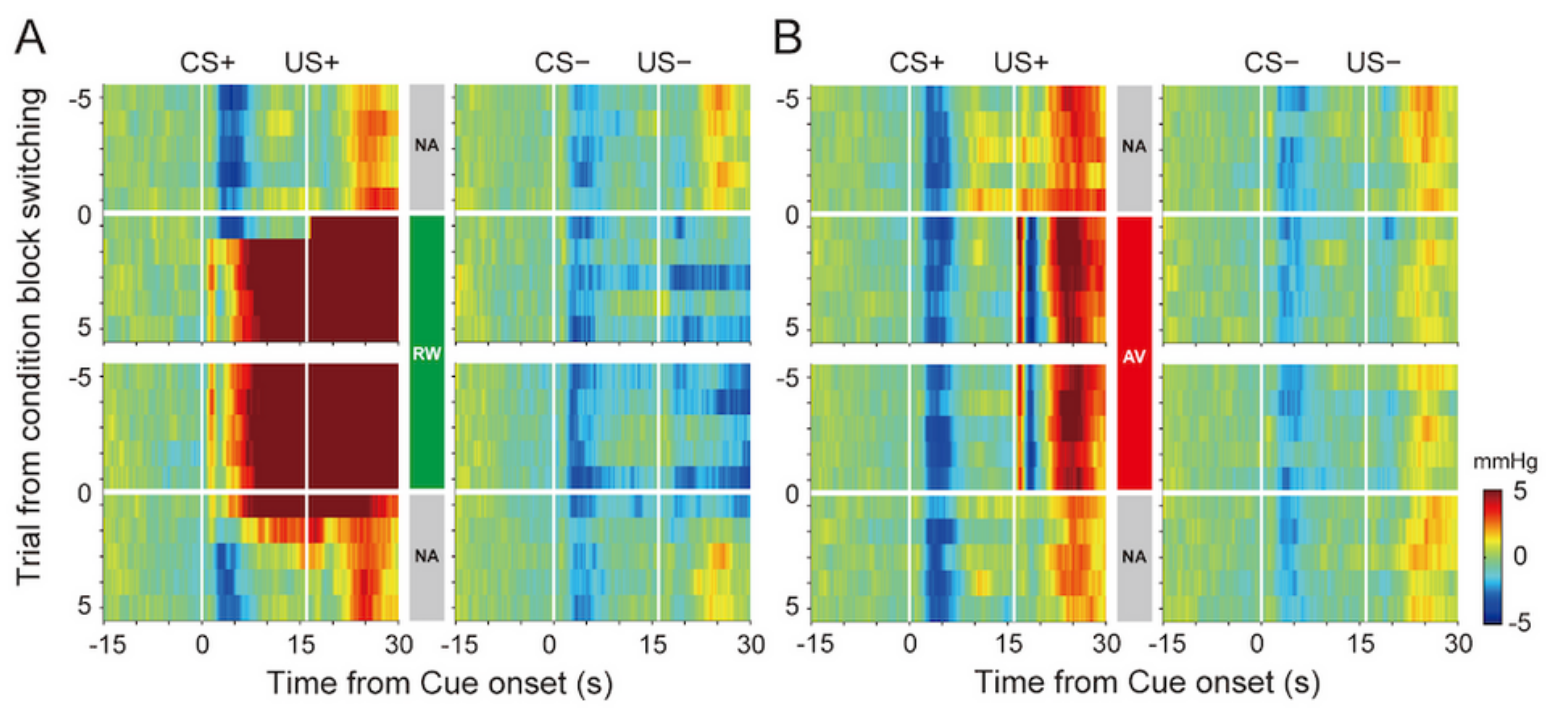

Figure 6

Dynamic changes of blood pressure responses triggered by condition block switching. Pseudo-color plots showing averaged mean blood pressure $(\triangle \mathrm{MBP})$ during five trials in each trial type CSs (left panels, CS+; right panels, CS-) before and after switching of reward condition block ( $A, N A \rightarrow R W, R W \rightarrow N A$, from upper to bottom) and aversive condition block ( $B, N A \rightarrow A V, A V \rightarrow N A$, from upper to bottom). $R W$, reward block; $\mathrm{NA}$, neutral block, AV, aversive block.

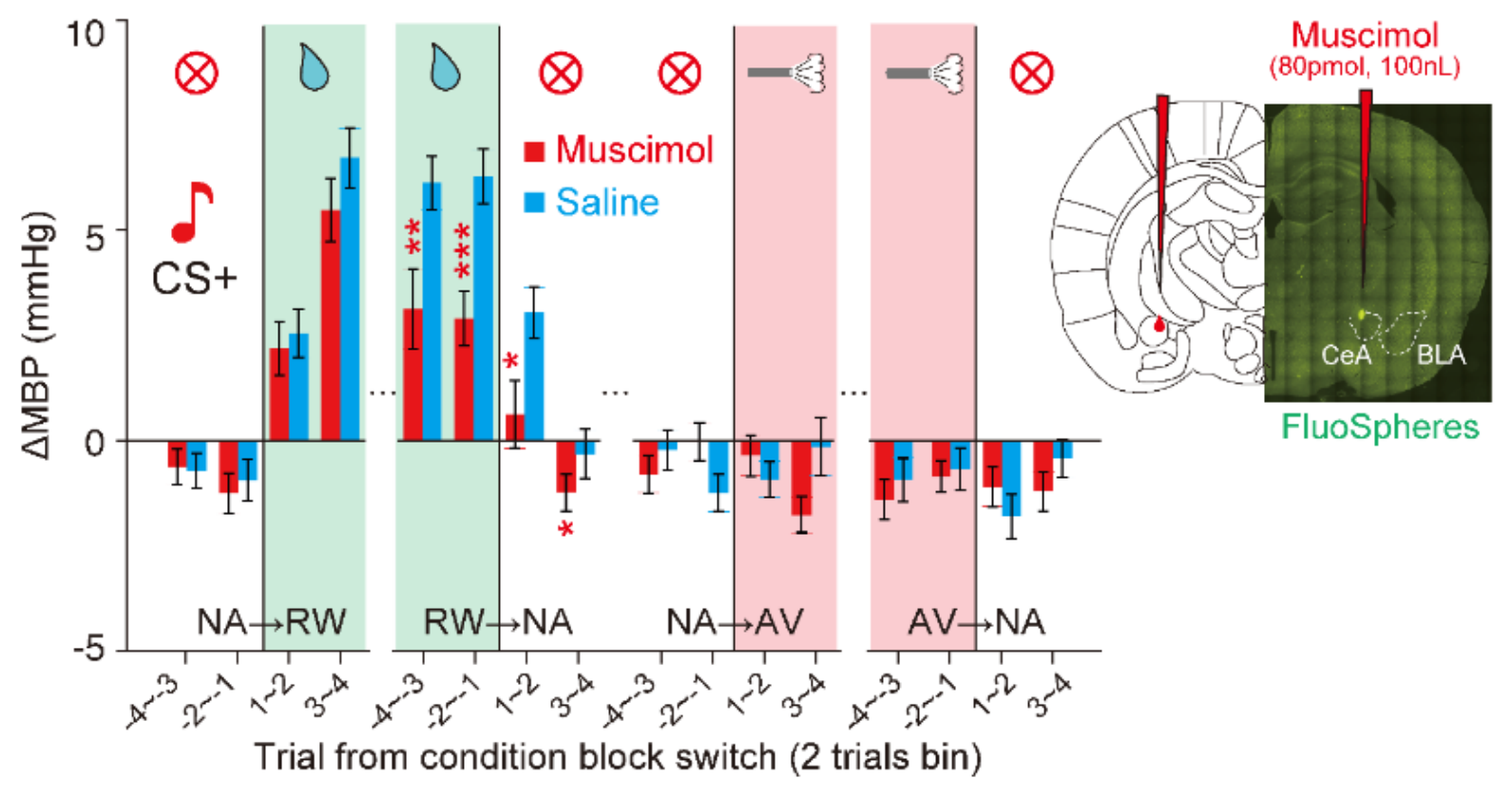

Figure 7 
Inactivation of bilateral $\mathrm{CeA}$ attenuated reward prediction-induced pressor response. Average data of mean blood pressure $(\triangle \mathrm{MBP})$ in response to $\mathrm{CS}+$ for the effects of inactivation of bilateral CeA before and after four trials (two trials per bin) at context block switching during the Pavlovian conditioning task (left panel). Red and cyan bars indicate the data of muscimol (12 injections from 4 rats) and saline (11 injections from 4 rats). ${ }^{*} p<0.05,{ }^{*} p<0.01,{ }^{* \star} p<0.001$, two-way ANOVA and Mann-Whitney $U$ test. The injection site of a GABAA agonist, muscimol ( $80 \mathrm{pmol} .100 \mathrm{~nL})$, in the bilateral CeA. Fluorospheres (100 $\mathrm{nL}$ ) were injected at stereotaxically identical positions using glass micro pipettes (right panel). CeA, central nucleus of the amygdala; BLA, basolateral amygdala. 